(C) 2020. This manuscript version is made available under the CC-BY-NC-ND 4.0 license http://creativecommons.org/licenses/by-nc-nd/4.0/

\title{
On admissible orders over closed subintervals of $[0,1]$
}

\author{
Fagner Santana ${ }^{\mathrm{a}, *}$, Benjamin Bedregal ${ }^{\mathrm{b}, \mathrm{c}}$, Petrucio Viana ${ }^{\mathrm{d}}$, Humberto \\ Bustince $^{\mathrm{c}, \mathrm{e}}$ \\ ${ }^{a}$ Departamento de Matemática, Universidade Federal do Rio Grande do Norte, Brazil \\ ${ }^{b}$ Departamento de Informática e Matemática Aplicada, Universidade Federal do Rio \\ Grande do Norte, Brazil \\ ${ }^{c}$ Institute of Smart Cities, Universidad Pública de Navarra, Spain \\ ${ }^{d}$ Departamento de Matemática e Estatística, Universidade Federal Fluminense, Brazil \\ ${ }^{e}$ Departamento de Estadística, Informática y Matemática, Universidad Pública de \\ Navarra, Spain
}

\begin{abstract}
In this paper, we make some considerations about admissible orders on the set of closed subintervals of the unit interval $\mathbb{I}[0,1]$, i.e., linear orders that refine the product order on intervals. We propose a new way to generate admissible orders on $\mathbb{I}[0,1]$ which is more general than those we find in the current literature. Also, we deal with the possibility of an admissible order on $\mathbb{I}[0,1]$ to be isomorphic to the usual order on $[0,1]$. We prove that some orders constructed by our method are not isomorphic to the usual one and we make some considerations about the question: is there some admissible order on $\mathbb{I}[0,1]$ isomorphic to the usual order on $[0,1]$ ?
\end{abstract}

Keywords: Interval-Valued Fuzzy Sets, Order Isomorphism, Admissible Order, Cantor's Bijection

\section{Introduction}

In [9] the concept of an admissible order on the set of closed subintervals of the unit interval was introduced. Admissible orders on $\mathbb{I}[0,1]=\{[a, b] \subseteq$ $[0,1]\}$, or just admissible orders when the context is clear, are linear orders

\footnotetext{
*Corresponding author.

Email addresses: fagner@ccet.ufrn.br (Fagner Santana), bedregal@dimap.ufrn.br (Benjamin Bedregal), petrucio_viana@id.uff.br (Petrucio Viana), bustince@unavarra.es (Humberto Bustince)
} 
that refine the product order $\leq_{2}$ on $\mathbb{I}[0,1]$, where $[a, b] \leq_{2}[c, d]$ iff $a \leq c$ and $b \leq d$. It is work of note that this order agree with the underlying order to the interval lattice generated by the lattice $\langle[0,1], \leq\rangle[20,21,31]$. Motivations for studying admissible orders comes from the theory of interval-valued fuzzy sets [33] and the fact that they find applications in areas such as image processing [8, 14], classification [27], clustering [29], decision making [10, 18], games [2], etc. If we think, as in [26], of an interval as representing uncertain information about a real number, the product order is a very natural extension of the usual order on $\mathbb{R}$ to the set of all intervals. Unfortunately, $\leq_{2}$ is not a linear order, e.g. $[0,1]$ and $[0.3,0.4]$ are not $\leq_{2}$-comparable, and this can be problematic in some applications. For example, in a simple clustering algorithm that uses interval data [30], we must set the objects to a cluster represented by the prototype of which it is nearest. If the algorithm uses interval distance $[25,30]$ as the similarity measure, than some distance values can be not comparable. For these situations, an admissible order could be useful, since intervals that are comparable by $\leq_{2}$ remain comparable in the same way by the admissible order and it can also deal with the remaining cases. In the first section, we propose a way to generate admissible orders which is more general that the one found in [9].

In [9], besides the introduction of the concept, a theoretical approach on admissible orders was started. From this seminal paper, some research on admissible orders had been made containing a range of theoretical contributions, such as $[1,10,34]$. Since both orders are linear and there exist bijections between $\mathbb{I}[0,1]$ and $[0,1]$, from a theoretical point of view, a natural question that arises is: are all the admissible orders isomorphic to the usual order on $[0,1]$ ? If the answer to this is yes, the theoretical approaches to these orders would be useless because all results on admissible orders would be simple adaptations of the ones on $[0,1]$ with its usual order. In the second section, we prove that some of the admissible orders constructed by the method we propose are not isomorphic to the usual order on $\mathbb{I}[0,1]$, including the lexicographical, antilexicographical, and Xu and Yager ([32]) orders. From this, we can conclude that the theory of admissible orders is not "empty", i.e., it is more than a simple rewriting of the theory of the usual order.

In the final section, we discuss the possibility of some admissible order to be isomorphic to the usual order on $[0,1]$. Our analysis is based on the Cantor-Schroeder-Bernstein theorem [5, 7, 11, 28]. 


\section{Generating admissible orders}

We denote by $\mathbb{I}[0,1]$ the set of all closed intervals with end-points lying in the interval $[0,1]$, i.e., $\mathbb{I}[0,1]=\{[a, b]: 0 \leq a \leq b \leq 1\}$; and by $\leq_{2}$ the product order on $\mathbb{I}[0,1]$, i.e., $[a, b] \leq_{2}[c, d] \Leftrightarrow a \leq c$ and $b \leq d$. Besides, we identify the sets $\mathbb{I}[0,1]$ and $\mathbb{K}[0,1]=\left\{(a, b) \in[0,1]^{2}: a \leq b\right\}$, via the trivial isomorphism.

In [9] the authors introduced the concept of admissible orders as linear orders in $\mathbb{I}[0,1]$ which refines the product order.

Example 1. The following relations are admissible orders:

- $[a, b] \leq_{\text {Lex } 1}[c, d] \Leftrightarrow(a<c) \vee[(a=c) \wedge(b \leq d)]$ (Lexicographical order)

- $[a, b] \leq_{\text {Lex } 2}[c, d] \Leftrightarrow(b<d) \vee[(b=d) \wedge(a \leq c)]$ (Antilexicographical order)

- $[a, b] \leq_{X Y}[c, d] \Leftrightarrow(a+b<c+d) \vee[(a+b=c+d) \wedge(b-a \leq d-c)]$ (Xu and Yager's order[32])

The concept of admissible order can be immediately generalized for orders in $[0,1]^{2}$, based on the product order in $[0,1]^{2}$. In this case, the three orders above (adapted to $[0,1]^{2}$ ) are admissible orders in $[0,1]^{2}$.

In the same paper $([9])$, they present a way to generate linear orders on $\mathbb{I}[0,1]$ which refines the product order on $\mathbb{I}[0,1]$, the so-called admissible orders, from aggregation functions. If $A, B:[0,1]^{2} \longrightarrow[0,1]$ are aggregation functions such that $A(x, y)=A(z, t)$ and $B(x, y)=B(z, t)$ if, and only if, $(x, y)=(z, t)$, then the relation $\leq_{A B}$ defined by:

$[x, y] \leq_{A B}[z, t] \Leftrightarrow(A(x, y)<A(z, t)) \vee(A(x, y)=A(z, t) \wedge B(x, y) \leq B(z, t))$

is an admissible order.

This order $\leq_{A B}$ is clearly based on the order $\leq_{L e x 1}$ combined with the functions $A$ and $B$. Based on this, we present the following result that shows ways to construct new admissible orders on $\mathbb{I}[0,1]$ from admissible orders on $[0,1]^{2}$ and functions $A, B: \mathbb{I}[0,1] \longrightarrow[0,1]$. For this, we will denote by $h_{A B}$ the function $h_{A B}: \mathbb{I}[0,1] \longrightarrow[0,1]^{2}$ defined by $h_{A B}([x, y])=$ $(A([x, y]), B([x, y]))$. 
Theorem 1. Let $A, B: \mathbb{I}[0,1] \longrightarrow[0,1]$ be functions and $\unlhd$ be an admissible order on $[0,1]^{2}$. The relation $\preceq_{A B \unlhd}$ defined by:

$$
[x, y] \preceq_{A B \unlhd}[z, t] \Leftrightarrow(A([x, y]), B([x, y])) \unlhd(A([z, t]), B([z, t]))
$$

is an admissible order on $\mathbb{I}[0,1]$ if, and only if, $h_{A B}$ is an injective and isotonic function between the posets $\left\langle\mathbb{I}[0,1], \leq_{2}\right\rangle$ and $\left\langle[0,1]^{2}, \unlhd\right\rangle$.

Proof. $(\Rightarrow)$ Suppose that $\preceq_{A B \unlhd}$ is an admissible order and take $[x, y],[z, t] \in$ $\mathbb{I}[0,1]$ such that $h_{A B}([x, y])=h_{A B}([z, t])$. So, $[x, y] \preceq_{A B \unlhd}[z, t]$ and $[z, t] \preceq_{A B \unlhd}$ $[x, y]$. Therefore, since $\preceq_{A B \unlhd}$ is an order, $[x, y]=[z, t]$. Whence, $h_{A B}$ is injective.

Now, suppose that $[x, y] \leq_{2}[z, t]$. Since $\preceq_{A B \unlhd}$ refines $\leq_{2}$, we have $[x, y] \preceq_{A B \unlhd}[z, t] \Leftrightarrow h_{A B}([x, y]) \unlhd h_{A B}([z, t])$. So, $h_{A B}$ is an isotonic function between $\left\langle\mathbb{I}[0,1], \leq_{2}\right\rangle$ and $\left\langle[0,1]^{2}, \unlhd\right\rangle$.

$(\Leftarrow)$ Suppose that $h_{A B}$ is injective and isotonic. The reflexivity of $\preceq_{A B \unlhd}$ is trivial.

For the antisymmetry, suppose that $[x, y] \preceq_{A B \unlhd}[z, t]$ and $[z, t] \preceq_{A B \unlhd}$ $[x, y]$. Thus, $(A([x, y]), B([x, y])) \unlhd(A([z, t]), B([z, t]))$ and $(A([z, t]), B([z, t])) \unlhd$ $(A([x, y]), B([x, y]))$, that is, $h_{A B}([x, y])=h_{A B}([z, t])$, which implies, by the injectivity of $h_{A B}$, that $[x, y]=[z, t]$.

The transitivity and linearity of $\preceq_{A B \unlhd}$ follow immediately from the transitivity and linearity of $\unlhd$.

Finally, suppose $[x, y] \leq_{2}[z, t]$. Since $h_{A B}$ is an isotonic function between $\left\langle\mathbb{I}[0,1], \leq_{2}\right\rangle$ and $\left\langle[0,1]^{2}, \unlhd\right\rangle$, we have $h_{A B}([x, y]) \unlhd h_{A B}([z, t]) \Leftrightarrow[x, y] \preceq_{A B \unlhd}$ $[z, t]$.

Observation 1. The admissible orders generated by aggregation functions in [9] are particular cases of the ones in Theorem 1 with $A$ and $B$ being aggregation functions in $[0,1]^{2}$ and taking as linear order $\leq_{\text {Lex } 1}$.

The next three corollaries present necessary and sufficient conditions for $h_{A B}$ satisfy the conditions in the above theorem when $\unlhd \in\left\{\leq_{L e x 1}, \leq_{L e x 2}\right.$ ,$\left.\leq_{X Y}\right\}$. Since the three proofs are analogous, we present just the case $\unlhd=\leq_{\text {Lex } 1}$ in detail.

Corollary 1. If $\unlhd=\leq_{L e x 1}$, then $h_{A B}$ is injective and isotonic if, and only if, $h$ is injective, $A$ is $\leq_{2}$-increasing and $B$ restricted to the set $\Gamma_{[a, b]}=\{[c, d] \in$ $\mathbb{I}[0,1]: A([a, b])=A([c, d])\}$ (note that $\Gamma_{[a, b]}=A^{-1}(A[a, b])$ ) is $\leq_{2}$-increasing for each $[a, b] \in \mathbb{I}[0,1]$. 
Proof. $(\Rightarrow)$ Suppose that $h_{A B}$ is an injective and isotonic function and $[x, y] \leq_{2}[z, t]$. Thus, $h_{A B}([x, y]) \leq_{L e x 1} h_{A B}([z, t])$, so $A([x, y])<A([z, t])$ or $A([x, y])=A([z, t])$ and $B([x, y]) \leq B([z, t])$, that is, we must have $A([x, y]) \leq A([z, t])$, which means that $A$ is $\leq_{2}$-increasing. Now, suppose that $[x, y],[z, t] \in \Gamma_{[a, b]}$ and $[x, y] \leq_{2}[z, t]$. We have $A([x, y])=A([z, t])=$ $A([a, b])$. Since $h_{A B}$ is isotonic, we have $h_{A B}([x, y]) \leq_{L e x 1} h_{A B}([z, t])$, so $(A([x, y]), B([x, y])) \leq_{\text {Lex } 1}(A([z, t]), B([z, t]))$ and since $A([x, y])=A([z, t])$, we have $B([x, y]) \leq B([z, t])$, that is, $B$ is $\leq_{2}$-increasing on $\Gamma_{[a, b]}$.

$(\Leftarrow)$ Suppose that $A$ is $\leq_{2}$-increasing, $B$ is $\leq_{2}$ increasing in each $\Gamma_{[a, b]}$ and $h_{A B}$ is injective. Take $[x, y],[z, t] \in \mathbb{I}[0,1]$ such that $[x, y] \leq_{2}[z, t]$. Thus, $h_{A B}([x, y]) \leq A([z, t])$. If $A([x, y])<A([z, t])$, then $(A([x, y]), A([x, y])) \leq_{\text {Lex } 1}$ $(A([z, t]), B([z, t]))$, so, $h_{A B}([x, y]) \leq_{L e x 1} h_{A B}([z, t])$. If $A([x, y])=A([z, t])$, then $[x, y],[z, t] \in \Gamma_{[x, y]}$, so $B([x, y]) \leq B([z, t]) \Rightarrow h_{A B}([x, y]) \leq_{L e x 1} h_{A B}([z, t])$.

Observation 2. There are functions $A$ and $B$ satisfying the conditions of the above corollary without $B$ being $\leq_{2}$-increasing. Take $A([x, y])=x$ and $B([x, y])=y-x$. If $A([x, y])=A([z, t])$, then $x=z$, so, if $[x, y] \leq_{2}[z, t]$, then $y \leq t \Rightarrow y-x \leq t-x=t-z \Rightarrow B([x, y]) \leq B([z, t])$. Clearly $h_{A B}$ is injective. Note that $B([0.1,0.2])=0.1$ and $B([0.15,0.2])=0.05$, so, $B$ is not $\leq_{2}$-increasing.

Example 2. With $A([x, y])=x, B([x, y])=y($ or $B([x, y])=y-x)$ and $\unlhd=\leq_{\text {Lex } 1}$ we have $\preceq_{A B \unlhd}=\leq_{\text {Lex } 1}$.

Corollary 2. If $\unlhd=\leq_{L e x 2}$, then $h_{A B}$ is injective and isotonic if, and only if, $h$ is injective, $B$ is $\leq_{2}$-increasing and $A$ restricted to the set $\Gamma_{[a, b]}^{\prime}=\{[c, d] \in$ $\mathbb{I}[0,1]: B([a, b])=B(c, d)\}$ is $\leq_{2}$-increasing for each $[a, b] \in \mathbb{I}[0,1]$.

Its immediate that if $A$ and $B$ are $\leq_{2}$-increasing and $h_{A B}$ is injective, then the conditions of the two corollaries hold.

Example 3. For $A([x, y])=y$ and $B([x, y])=\frac{x+y}{2}$, we have $\preceq_{A B \leq_{L e x 1}}=\leq_{\text {Lex } 2}$ and $\preceq_{A B \leq_{\text {Lex } 2}}=\leq_{\text {Lex } 1}$.

Corollary 3. If $\unlhd=\leq_{X Y}$, then $h_{A B}$ is injective and isotonic if, and only if, $h$ is injective, the function $f([x, y])=A([x, y])+B([x, y])$ is $\leq_{2}$-increasing and the function $g([x, y])=B([x, y])-A([x, y])$ restricted to the set $\Gamma_{[a, b]}^{\prime \prime}=$ $\{[c, d] \in \mathbb{I}[0,1]: f([a, b])=f([c, d])\}$ is $\leq_{2}$-increasing for each $[a, b] \in \mathbb{I}[0,1]$. 
Observation 3. If $A$ and $B$ are $\leq_{2}$-increasing and $h$ is injective, then the conditions of the Corollary 3 hold. In fact, in this case $f([x, y])=A([x, y])+$ $B([x, y])$ is trivially $\leq_{2}$-increasing. Now, suppose that $g([x, y])=B([x, y])-$ $A([x, y])$ is not $\leq_{2}$-increasing in some $\Gamma_{[a, b]}^{\prime \prime}$ and take $[x, y],[z, t] \in \Gamma_{[a, b]}^{\prime \prime}$ such that $[x, y] \leq_{2}[z, t]$ but $g([x, y])>g([z, t]) \Leftrightarrow B([x, y])-A([x, y])>B([z, t])-$ $A([z, t])$. Since $[x, y],[z, t] \in \Gamma_{[a, b]}^{\prime \prime}$, we have $A([x, y])+B([x, y])=A([z, t])+$ $B([z, t])$, so $2 B([x, y])>2 B([z, t]) \Rightarrow B([x, y])>B([z, t])$, which contradicts $B$ to be $\leq_{2}$-increasing.

Example 4. Take $A([x, y])=y, B([x, y])=\frac{x+y}{2}$ and $\unlhd=\leq_{X Y}$. Note that $h_{A B}([x, y])=h_{A B}([z, t]) \Leftrightarrow y=t$ and $\frac{x+y}{2}=\frac{z+t}{2}$, so $x=z$ and $h_{A B}$ is injective. Since $A$ and $B$ are $\leq_{2}$-increasing $\preceq_{A B \leq_{X Y}}$ is an admissible order. Note that:

- $\preceq_{A B \leq_{X Y}} \neq \leq_{X Y}:[0.15,0.17] \preceq_{A B \leq_{X Y}}[0.1,0.2]$ but $[0.1,0.2] \leq_{X Y}[0.15,0.17]$;

- $\preceq_{A B \leq_{X Y}} \neq \leq_{L e x 1}:[0.15,0.17] \preceq_{A B \leq_{X Y}}[0.1,0.2]$ but $[0.1,0.2] \leq_{\text {Lex } 1}[0.15,0.17]$;

- $\preceq_{A B \leq_{X Y}} \neq \leq_{\text {Lex2 } 2}:[0.15,0.17] \preceq_{A B \leq_{X Y}}[0.16,0.169]$ but $[0.16,0.169] \leq_{\text {Lex } 2}$ $[0.15,0.17]$.

\section{Relation between some admissible orders and the chain $\langle[0,1], \leq\rangle$}

In this section we discuss the possibility of an admissible order being isomorphic to the usual order on $[0,1]$, that is, the existence of an order isomorphism between $\langle\mathbb{I}[0,1], \preceq\rangle$, where $\preceq$ is an admissible order, and $\langle[0,1], \leq\rangle$.

In a poset $\left\langle C, \leq_{C}\right\rangle$, given $a \leq_{C} b$ we define the set $[a, b]_{\leq_{C}}=\{x \in C$ : $\left.a \leq_{C} x \leq_{C} b\right\}$, i.e., this is the closed interval, w.r.t. $\leq_{C}$, with end-points $a$ and $b$. This interval will be called non-degenerated if $a<_{C} b$. Note that an interval $[a, b]_{\leq C}$ is never empty.

Observation 4. If $\left\langle C, \leq_{C}\right\rangle$ and $\left\langle D, \leq_{D}\right\rangle$ are posets such that $\leq_{C}$ and $\leq_{D}$ are linear orders and $f: C \longrightarrow D$ is a bijection that preserves order (an order isomorphism between $\left\langle C, \leq_{C}\right\rangle$ and $\left.\left\langle D, \leq_{D}\right\rangle\right)$, then it is immediate to note that for all $x, y \in C$ with $x \leq_{C} y$, we have $f\left([x, y]_{\leq_{C}}\right)=[f(x), f(y)]_{\leq_{D}}$. Besides that, if $[x, y]_{\leq_{C}}$ is a non-degenerated interval, then $f\left([x, y]_{\leq_{C}}\right)$ is also a non-degenerated interval. 
The following basic lemma is a well known result of Mathematical Analysis (see [24]).

Lemma 1. Every family of non-degenerated pairwise disjoint intervals of $\mathbb{R}$ is countable.

In the next results we prove that some classes of admissible orders are not isomorphic to the usual order on $[0,1]$. For this, its enough to construct an uncountable family of non-degenerated intervals in the poset $\langle\mathbb{I}[0,1], \preceq\rangle$, so, by the Observation 4 , if there were an isomorphism between this poset and $\langle[0,1], \leq\rangle$, we would have an uncountable family of non-degenerated intervals on $\langle[0,1], \leq\rangle$, which contradicts Lemma 1 .

Theorem 2. Let $A, B: \mathbb{I}[0,1] \longrightarrow[0,1]$ be functions such that $\preceq_{A B \leq_{L e x 1}}$ is an admissible order. Suppose there are $t_{1}, t_{2} \in[0,1]$, with $t_{1}<t_{2}$ such that $A([x, y])=x$, for all $x \in\left(t_{1}, t_{2}\right)$. Thus, $\left\langle\mathbb{I}[0,1], \preceq_{A B \leq_{\text {Lex } 1}}\right\rangle$ is not isomorphic to $\langle[0,1], \leq\rangle$.

Proof. For each $x \in\left(t_{1}, t_{2}\right)$, consider the intervals $[x, x]$ and $[x, 1]$. Since $A([x, x])=A([x, 1])=x$ and $[x, x] \leq_{2}[x, 1]$, we have $[x, x],[x, 1] \in \Gamma_{[x, x]}$, so, by corollary $1, B([x, x])<B([x, 1])\left(h_{A B}\right.$ is injective), so $[x, x]<_{A B \leq_{\text {Lex } 1}}$ $[x, 1]$. For each $x \in\left(t_{1}, t_{2}\right)$ define $I_{x}=[[x, x],[x, 1]]_{\preceq_{A B \leq L e x 1}}$. Note that $I_{x}$ is a non-degenerated interval and if $[a, b] \in I_{x}$, then $A([a, b])=x$, so, if $x, y \in\left(t_{1}, t_{2}\right)$, with $x \neq y$, then $I_{x} \cap I_{y}=\emptyset$, which implies that the family $\left\{I_{x}\right\}_{x \in\left(t_{1}, t_{2}\right)}$ is an uncountable family of non-degenerated intervals and this is enough to conclude the non-existence of the isomorphism.

Example 5. If $A([x, y])=x$ and $B([x, y])=y$, then $\preceq_{A B \leq_{L e x 1}}=\leq_{L e x 1}$, so $\leq_{\text {Lex } 1}$ is not isomorphic to the usual order on $[0,1]$.

Example 6. Consider $A([x, y])=\left\{\begin{array}{cl}x & , \text { if } 0 \leq x \leq 1 / 2 \\ \frac{x+y}{2} & , \text { if } 1 / 2<x \leq 1\end{array}\right.$ and $B([x, y])=$ $y-x$. Suppose that $A([x, y])=A([z, t])$, so $x, z \leq 1 / 2$ or $x, z>1 / 2$. In the first case $A([x, y])=x=A([z, t])=z \Rightarrow x=z$. So, if $B([x, y])=B([z, t])$, then $y-x=t-z \Rightarrow y=z$. If $x, z>1 / 2$, then $x+y=z+t$ and if $B\left(([x, y])=B([z, t])\right.$, then $y-x=t-z$, so $y=t$ and $x=y$. Thus, $h_{A B}$ is injective. Besides that, $A$ is clearly $\leq_{2}$-increasing and if $A([x, y])=A([z, t])$ with $[x, y] \leq_{2}[z, t]$, then for the case $x, z \leq 1 / 2$, we have $x=z$, so, from 
$y \leq t$ it follows that $y-x \leq t-z$, that is, $B([x, y]) \leq B([z, t])$ and for the case $x, z>1 / 2$, we have $x+y=z+t$. Suppose that $y-x>t-z$. Thus, $2 y>2 t \Rightarrow y>t$, which is a contradiction. So, $B$ is $\leq_{2}$-increasing in $\Gamma_{[a, b]}$, for all $[a, b] \in \mathbb{I}[0,1]$. We can conclude that $\preceq_{A B \leq_{\text {Lex } 1}}$ is an admissible order and, for $t_{1}=0$ and $t_{2}=1 / 2$, the theorem 2 ensures that this order is not isomorphic to the usual order on $[0,1]$. Besides, this order is different from the orders already seen:

- $\preceq_{A B \leq_{\text {Lex } 1}} \neq \leq_{X Y}:[0,0.4] \preceq_{A B \leq_{\text {Lex } 1}}[0.05,0.05]$ but $[0.05,0.05] \leq_{X Y}[0,0.4]$;

- $\preceq_{A B \leq_{\text {Lex } 1}} \neq \leq_{\text {Lex } 1}:[0.6,0.62] \preceq_{A B \leq_{\text {Lex } 1}}[0.54,0.7]$ but $[0.54,0.7] \leq_{\text {Lex } 1}$ $[0.6,0.62]$;

- $\preceq_{A B \leq_{\text {Lex } 1}} \neq \leq_{\text {Lex } 2}:[0.51,0.71] \preceq_{A B \leq_{\text {Lex } 1}}[0.54,0.7]$ but $[0.54,0.7] \leq_{\text {Lex } 2}$ $[0.51,0.71]$.

Theorem 3. Let $A, B: \mathbb{I}[0,1] \longrightarrow[0,1]$ be functions such that $\preceq_{A B \leq_{\text {Lex } 2}}$ is an admissible order. Suppose there are $t_{1}, t_{2} \in[0,1]$, with $t_{1}<t_{2}$ such that $B([x, y])=y$, for all $y \in\left(t_{1}, t_{2}\right)$. Thus, $\left\langle\mathbb{I}[0,1], \preceq_{A B \leq_{\text {Lex } 2}}\right\rangle$ is not isomorphic to $\langle[0,1], \leq\rangle$.

PROOF. For each $y \in\left(t_{1}, t_{2}\right)$, consider the interval $I_{y}=[[0, y],[y, y]]_{\preceq_{A B \leq} \leq_{\text {Lex } 2}}$. Since $y>0$, we have $[0, y]<_{2}[y, y]$, so the interval $I_{y}$ is non-degenerated, for all $y \in\left(t_{1}, t_{2}\right)$. If $[a, b] \in I_{y}$, then $B([a, b])=y$, so if $x, y \in\left(t_{1}, t_{2}\right)$, with $x \neq y$, then $I_{x} \cap I_{y}=\emptyset$, which implies that the family $\left\{I_{x}\right\}_{x \in\left(t_{1}, t_{2}\right)}$ is an uncountable family of non-degenerated intervals.

Example 7. If $A([x, y])=x$ and $B([x, y])=y$, then $\preceq_{A B \leq_{\text {Lex } 2}}=\leq_{\text {Lex } 2}$, so $\leq_{\text {Lex } 2}$ is not isomorphic to the usual order on $[0,1]$.

Example 8. Consider $A([x, y])=\left\{\begin{array}{cc}\frac{x+y}{2} & , \text { if } 0 \leq y \leq 1 / 2 \\ y & , \text { if } 1 / 2<y \leq 1\end{array}\right.$ and $B([x, y])=$ $y-x$. With this two functions, $\preceq_{A B \leq_{L e x 2}}$ is an admissible order which is different from the orders $\leq_{\text {Lex } 1}, \leq_{\text {Lex } 2}, \leq_{X Y}$, and the order from Example 6 . Besides, for $t_{1}=1 / 2$ and $t_{2}=1$, Theorem 3 ensures that this order is not isomorphic to the usual order on $[0,1]$.

Theorem 4. Let $A, B: \mathbb{I}[0,1] \longrightarrow[0,1]$ be functions such that $\preceq_{A B \leq_{X Y}}$ is an admissible order. Suppose there are $t_{1}, t_{2} \in[0,1]$, with $t_{1}<t_{2}$ such that $A([x, y])=x$ and $B([x, y])=y$, for all $x \in\left(t_{1}, t_{2}\right)$. Thus, $\left\langle\mathbb{I}[0,1], \preceq_{A B \leq_{X Y}}\right\rangle$ is not isomorphic to $\langle[0,1], \leq\rangle$. 
Proof. For each $x \in\left(t_{1}, t_{2}\right)$, consider the intervals $[x / 2, x / 2]$ and $[0, x]$. Since $A([x / 2, x / 2])+B([x / 2, x / 2])=A([0, x])+B([0, x])=x$ and $B([x / 2, x / 2]-$ $A([x / 2, x / 2])=0<x / 2=B([0, x])-A([0, x])=x$, we have $[x / 2, x / 2] \prec_{A B \leq_{X Y}}$ $[0, x]$. Define $I_{x}=[[x / 2, x / 2],[0, x]]_{\preceq_{A B}<_{X Y}}$ for each $x \in\left(t_{1}, t_{2}\right)$. If $[a, b] \in \bar{I}_{x}$, then $A([a, b])+B([a, b])=x$, so, if $x, y \in\left(t_{1}, t_{2}\right)$, with $x \neq y$, then $I_{x} \cap I_{y}=\emptyset$, which implies that the family $\left\{I_{x}\right\}_{x \in\left(t_{1}, t_{2}\right)}$ is an uncountable family of nondegenerated intervals.

Example 9. If $A([x, y])=x$ and $B([x, y])=y$, then $\preceq_{A B \leq_{X Y}}=\leq_{X Y}$, so $\leq_{X Y}$ is not isomorphic to the usual order on $[0,1]$.

Example 10. Consider $A([x, y])=\left\{\begin{array}{cl}x & , \text { if } 0 \leq x \leq 1 / 2 \\ \frac{x+y}{2} & , \text { if } 1 / 2<x \leq 1\end{array}\right.$ and $B([x, y])=$ $y$. With this two functions, $\preceq_{A B \leq_{X Y}}$ is an admissible order which is different from the orders $\leq_{L e x 1}, \leq_{L e x 2}, \leq_{X Y}$, and from the orders in Examples 6 and 8. Besides, for $t_{1}=0$ and $t_{2}=1 / 2$, Theorem 4 ensures that this order is not isomorphic to the usual order on $[0,1]$.

These last results and examples show a lot of admissible orders that are not isomorphic to the usual order on $[0,1]$. In fact, the unique possibility that we have for $\langle\mathbb{I}[0,1], \preceq\rangle$ be isomorphic to $\langle[0,1], \leq\rangle$ is with a non continuous isomorphism, where the continuity is inherited from $\mathbb{R}^{2}$ continuity when we consider an interval as a pair of numbers, i.e. $\mathbb{I}[0,1] \approx\left\{([a, b]) \in[0,1]^{2}: a \leq\right.$ $b\}$.

\subsection{An illustrative example for the use of admissible order in decision mak- ing}

The use of fuzzy sets in decision making problems is very common $([4,10$, 18]. The problem can be summarized as follows. There is a set of $n$ alternatives, say $\left\{x_{1}, \ldots, x_{n}\right\}$. The expert on the subject provides his/her preferences for each alternative over the others by using a fuzzy binary relation $R$ on $X$, that is, a function $R: X \times X \longrightarrow[0,1]$, where the value $R\left(x_{i}, x_{j}\right)=R_{i j}$ indicates the degree of preference of $x_{i}$ over $x_{j}$. When $R_{i j}+R_{j i}=1$, we say that the fuzzy relation $R$ have the property of reciprocity. The relation $R$ can be represented by the matrix

$$
R=\left(\begin{array}{cccc}
0 & R_{12} & \cdots & R_{1 n} \\
R_{21} & 0 & \cdots & R_{2 n} \\
\vdots & \vdots & \ddots & \vdots \\
R_{n 1} & R_{n 2} & \cdots & 0
\end{array}\right) .
$$


In reciprocal relations it is usual to not define the elements in the diagonal or take a fixed value. We opt for the value 0 .

Considering the relation $R$ as the preferences indicated by the expert, one of the most used method to make the choice among the alternatives is the weighted vote (see [16]), where the alternative chosen is $x_{k}$, where $k$ is the solution of:

$$
\arg \max _{i=1, \ldots, n} \sum_{1 \leq j \leq n} R_{i j}
$$

When the expert assigns the degree of preference of an alternative over other, the lack of knowledge of this expert is not taken into consideration and this fact may result in a lack of accuracy in choosing (see [3]). The concept of weak ignorance function was defined in [27] to measure this lack of knowledge.

Definition 1. (Sanz et al. [27]) A function $g:[0,1] \longrightarrow[0,1]$ is said to be a weak ignorance function if it is continuous and:

1. $g(x)=g(1-x)$, for all $x \in[0,1]$;

2. $g(x)=0$ if and only if $x=0$ or $x=1$;

3. $g(0.5)=1$.

Example 11. ([22]) $g(x)=2 \min (x, 1-x)$ is a weak ignorance function.

Given an ignorance function $g$ we define the map $g^{*}:[0,1] \rightarrow \mathbb{I}[0,1]$ for each $x \in[0,1]$ as follows:

$$
g^{*}(x)=[x(1-g(x)), x(1-g(x))+g(x)]
$$

Observe that if $g$ is such that $g(x)=g(y) \Leftrightarrow x \in\{y, 1-y\}$, as is the case of the $g$ in Example 11, then $g^{*}$ is injective but never will be bijective. In addition, $g^{*}(0.5)=[0,1], g^{*}(0)=[0,0]$ and $g^{*}(1)=[1,1]$.

In the decision making process mentioned above, the value $g\left(R_{i j}\right)$, where $g$ is a weak ignorance function, represents the ignorance in the preference value assigned by the expert of the alternative $x_{i}$ over $x_{j}$. Using some weak ignorance function $g$, we can represents the uncertainty that comes from ignorance by using intervals. Define $r_{i j}=g^{*}(\operatorname{Rij})$ (see $\left.[3,4,22]\right)$. Thus, $r$ is an interval-valued fuzzy binary preference relation (IVFBPR). 
As an example, consider the decision making problem with 4 alternatives $\left(\left\{x_{1}, x_{2}, x_{3}, x_{4}\right\}\right)$ where the preference fuzzy relation is given by:

$$
R=\left(\begin{array}{cccc}
0 & 0.31 & 0.16 & 0.36 \\
0.69 & 0 & 0.05 & 0.06 \\
0.84 & 0.95 & 0 & 0.38 \\
0.64 & 0.94 & 0.62 & 0
\end{array}\right)
$$

For this example, the weighted vote method returns that the alternative chosen is $x_{4}$. Using the function $g$ in Example 11, the IVFBPR is:

$$
r=\left(\begin{array}{cccc}
0 & {[0.1178,0.7378]} & {[0.1088,0.4288]} & {[0.1088,0.8208]} \\
{[0.2622,0.8822]} & 0 & {[0.045,0.145]} & {[0.0528,0.1728]} \\
{[0.5712,0.8912]} & {[0.855,0.955]} & 0 & {[0.0912,0.8512]} \\
{[0.1792,0.8992]} & {[0.8272,0.9472]} & {[0.1488,0.9088]} & 0
\end{array}\right)
$$

To choose the alternative we use the weighted vote method but adapted for the interval case, by using the usual sum of intervals and some admissible order. For this, we need to calculate $\sum_{1 \leq j \leq 4} r_{i j}, i \in\{1, \ldots, 4\}$ :

- $\sum_{1 \leq j \leq 4} r_{1 j}=[0.3274,1.9874]=I_{1}$

- $\sum_{1 \leq j \leq 4} r_{2 j}=[0.36,1.2]=I_{2}$;

- $\sum_{1 \leq j \leq 4} r_{3 j}=[1.5174,2.6974]=I_{3}$

- $\sum_{1 \leq j \leq 4} r_{4 j}=[1.1552,2.7552]=I_{4}$.

Since the admissible orders are defined in the set $\mathbb{I}[0,1]$, we normalize the intervals dividing each endpoint by 3 (the maximum value for the endpoints for these intervals). Thus, we obtain:

- $I_{1}^{\prime}=[0.109133,0.662466]$;

- $I_{2}^{\prime}=[0.12,0.4]$;

- $I_{3}^{\prime}=[0.5058,0.899133]$; 
- $I_{4}^{\prime}=[0.385066,0.9184]$.

By using the functions $A$ e $B$ of Example 6 , where the order $\preceq_{A B \leq_{L e x 1}}$ was defined, we have:

- $\left(A\left(I_{1}^{\prime}\right), B\left(I_{1}^{\prime}\right)\right)=(0.109133,0.5533)$;

- $\left(A\left(I_{2}^{\prime}\right), B\left(I_{2}^{\prime}\right)\right)=(0.12,0.28)$;

- $\left(A\left(I_{3}^{\prime}\right), B\left(I_{3}^{\prime}\right)\right)=(0.7024665,0.3933)$;

- $\left(A\left(I_{3}^{\prime}\right), B\left(I_{3}^{\prime}\right)\right)=(0.385066,0.53334)$.

Thus, by the order $\preceq_{A B \leq_{L e x 1}}$, the alternative $x_{3}$ is the chosen one.

By using the functions $A$ e $B$ of Example 8, where the order $\preceq_{A B \leq_{L e x 2}}$ was defined, we have:

- $\left(A\left(I_{1}^{\prime}\right), B\left(I_{1}^{\prime}\right)\right)=(0.662466,0.553333)$;

- $\left(A\left(I_{2}^{\prime}\right), B\left(I_{2}^{\prime}\right)\right)=(0.26,0.28)$;

- $\left(A\left(I_{3}^{\prime}\right), B\left(I_{3}^{\prime}\right)\right)=(0.899133,0.393333)$;

- $\left(A\left(I_{3}^{\prime}\right), B\left(I_{3}^{\prime}\right)\right)=(0.91984,0.53334)$.

Thus, by the order $\preceq_{A B \leq_{\text {Lex }} 2}$, the alternative $x_{1}$ is the chosen one.

By using the functions $A$ e $B$ of Example 10 where the order $\preceq_{A B \leq_{X Y}}$ was defined, we have:

- $\left(A\left(I_{1}^{\prime}\right), B\left(I_{1}^{\prime}\right)\right)=(0.109133,0.662466)$;

- $\left(A\left(I_{2}^{\prime}\right), B\left(I_{2}^{\prime}\right)\right)=(0.12,0.4)$;

- $\left(A\left(I_{3}^{\prime}\right), B\left(I_{3}^{\prime}\right)\right)=(0.7024665,0.899133)$;

- $\left(A\left(I_{3}^{\prime}\right), B\left(I_{3}^{\prime}\right)\right)=(0.385066,0.9184)$.

Thus, by the order $\preceq_{A B \leq_{X Y}}$, the alternative $x_{3}$ is the chosen one.

As could be seen, each order resulted in a different choice among the alternatives, only alternative $x_{2}$ was not chosen in either case. 


\section{Isomorphism: the general case}

We don't have the answer to the conjecture "If $\preceq$ is an admissible order on $\mathbb{I}[0,1]$, then $\langle\mathbb{I}[0,1], \preceq$,$\rangle is not isomorphic to \langle[0,1], \leq\rangle$ ", but we have some considerations about it, based on the following famous theorem:

Theorem 5 (Cantor-Schröder-Bernstein). Let $A$ and $B$ be non-empty sets. If there exist injections from $A$ to $B$ and from $B$ to $A$, then there exists a bijection between $A$ and $B$.

First of all, if there is a bijection $f: \mathbb{I}[0,1] \longrightarrow[0,1]$ such that $[a, b] \leq_{2}$ $[c, d] \Rightarrow f([a, b]) \leq f([c, d])$, then we can define the order $\leq_{f}$ by $[a, b] \leq_{f}$ $[c, d] \Leftrightarrow f([a, b]) \leq f([c, d])$. This order is clearly admissible and the function $f$ is an isomorphism between $\left\langle\mathbb{I}[0,1], \leq_{f}\right\rangle$ and $\langle[0,1], \leq\rangle$. The converse is trivially true, so, the original conjecture can be rewritten as "There is no bijection from $\mathbb{I}[0,1]$ to $[0,1]$ such that $[a, b] \leq_{2}[c, d] \Rightarrow f([a, b]) \leq f([c, d])$."

There are several proofs of the Cantor-Schröder-Bernstein Theorem [15]. Most of them have an existential flavor, that is, they guarantees the existence of the bijection but do not exhibit or build it. Following the ideas in [6] and [23], a constructible proof of this theorem is presented in [19]. The basic idea of this proof is constructing, from the injections $f: A \longrightarrow B$ and $g: B \longrightarrow A$, two sequences of sets $A_{n}$ and $B_{n}$

i) $A_{0}=A \backslash g(B)$;

ii) $B_{n}=f\left(A_{n}\right)$, for $n \in \mathbb{N}$;

iii) $A_{n}=g\left(B_{n-1}\right)$, for $n \in \mathbb{N} \backslash\{0\}$.

If $g$ is not surjective (if $g$ is surjective, $g^{-1}$ is, itself, the claimed bijection), then $A_{0}$ is non empty and, so, none of the sets in both sequences is empty. After, is proved that the function $H: A \longrightarrow B$ defined by:

$$
H(x)= \begin{cases}\tilde{f}(x) & , \text { if } x \in \bigcup_{n \in \mathbb{N}} A_{n} \\ \tilde{g}^{-1}(x) & , \text { if } x \in A \backslash \bigcup_{n \in \mathbb{N}} A_{n}\end{cases}
$$

where $\tilde{f}$ is a bijection from $\bigcup_{n} A_{n}$ to $\bigcup_{n} B_{n}$, obtained by restricting $f$ to $\bigcup_{n} A_{n}$ and, similarly, $\tilde{g}$ is a bijection which from $B \backslash \bigcup_{n} B_{n}$ to $A \backslash \bigcup_{n} A_{n}$, obtained by restricting $g$ to $\bigcup_{n} B_{n}$, is well defined and, finally, that $H$ is a bijection from $A$ to $B$. Unfortunately, this theorem does not involve orders. 
In fact, as will be seen, it is possible that the two injections $f$ and $g$ preserve orders but the function $H$ is not an order isomorphism. Below, we will present injections $f: \mathbb{I}[0,1] \longrightarrow[0,1]$ and $g:[0,1] \longrightarrow \mathbb{I}[0,1]$ that preserve the orders $\leq_{2}$ and $\leq$ but the function $H$ from the theorem is not an isomorphism. There are many injections $g$ that preserve the orders above. For example, $g(t)=[t, t]$, or $g(t)=[0, t]$, or $g(t)=[t, 1]$. To define an injection $f$ that preserve the orders, consider the infinite proper decimal expansion of the numbers in $[0,1)$ and consider the notation $0 . t_{1} t_{2} t_{3} \ldots$ for $t \in[0,1$ ) (there is no expansions such that $t_{k}=9$ for all $k \geq k_{0}$, for some $k_{0}$ ). If $t$ has a finite decimal expansion we consider $t_{k}=0$, for all $k$ not appearing in the expansion.

Proposition 1. The function $f: \mathbb{I}[0,1] \longrightarrow[0,1]$ defined by:

$$
f([x, y])= \begin{cases}0 . x_{1} y_{1} x_{2} y_{2} x_{3} y_{3} \ldots & , \text { if } 0 \leq x \leq y<1 \\ 0 . x_{1} 9 x_{2} 9 x_{3} 9 \ldots & , \text { if } 0 \leq x<y=1 \\ 1 & , \text { if } x=y=1\end{cases}
$$

is an injection such that $[a, b] \leq_{2}[c, d] \Rightarrow f([a, b]) \leq f([c, d])$.

Proof. The injectivity is immediate (see [19]). Suppose $[a, b] \leq_{2}[c, d]$, that is, $a \leq c$ and $b \leq d$, and denote $z=f([a, b])$ and $w=f([c, d])$. First, consider the case $a<c$ and $b=d<1$. Thus, there exists $k_{0} \in \mathbb{N}$ such that $a_{k}=c_{k}$ for all $k<k_{0}$ and $a_{k_{0}}<c_{k_{0}}$ and $b_{k}=d_{k}$ for all $k \in \mathbb{N}$. Thus, $z=0 . a_{1} b_{1} a_{2} b_{2} \ldots a_{k_{0}} b_{k_{0}} \ldots$ and $w=0 . c_{1} d_{1} c_{2} d_{2} \ldots c_{k_{0}} d_{k_{0}} \ldots$, so $z_{k}=w_{k}$ for all $k<2 k_{0}-1$ and $z_{2 k_{0}-1}=a_{k_{0}}<c_{k_{0}}=w_{2 k_{0}-1}$, which implies $z<w$. The case $a=c$ and $b<d$ is entirely analogous. Consider $a<c$ and $b<d$. There exists $k_{1}$ and $k_{2}$ such that $a_{k}=c_{k}$ for all $k<k_{1}$ and $a_{k_{1}}<c_{k_{1}}, b_{k}=d_{k}$ for all $k<k_{2}$ and $b_{k_{2}}<d_{k_{2}}$. Taking $k_{0}=\min \left\{k_{1}, k_{2}\right\}$, we have $z_{k}=w_{k}$ for all $k<2 k_{0}$ and $z_{2 k_{0}}<w_{2 k_{0}}$, or, $z_{2 k_{0}}=w_{2 k_{0}}$ and $z_{2 k_{0}-1}<w_{2 k_{0}-1}$ so $z<w$. The remaining cases, $d=1, b=d=1, c=d=1, b=c=d=1$ and $a=b=c=d=1$ are immediate or follows the same idea employed in the previous cases.

This function $f$ is not surjective. Indeed, $0 . x_{1} x_{2} x_{3} x_{4} x_{5} \ldots \notin \operatorname{Im}(f)$ if $0 . x_{1} x_{3} x_{5} \ldots \not \leq 0 . x_{2} x_{4} x_{6} \ldots$

Now, we prove that the bijection $H$ built from the the Cantor-SchröderBernstein Theorem, starting from the functions $f$ above and $g(t)=[t, t]$, is not isotonic, w.r.t. $\leq_{2}$ and $\leq$. We have: 
- $A_{0}=\mathbb{I}[0,1] \backslash g([0,1])=\{[x, y]: 0 \leq x<y \leq 1\}$

- $B_{0}=f\left(A_{0}\right)=\left\{0 . x_{1} x_{2} x_{3} \ldots: 0 . x_{1} x_{3} x_{5} \ldots \leq 0 . x_{2} x_{4} x_{6} \ldots\right.$ and $x_{2 k-1} \neq x_{2 k}$, for some $\left.k\right\}$;

- $A_{1}=\left\{[x, x]: x \in B_{0}\right\}$;

- $B_{1}=f\left(A_{1}\right)=\left\{0 . x_{1} x_{1} x_{2} x_{2} x_{3} x_{3} \ldots: 0 . x_{1} x_{3} x_{5} \ldots \leq 0 . x_{2} x_{4} x_{6} \ldots\right.$ and $x_{2 k-1} \neq x_{2 k}$, for some $\left.k\right\}$;

- $A_{2}=\left\{[x, x]: x \in B_{1}\right\}$;

- $B_{2}=f\left(A_{2}\right)=\left\{0 . x_{1} x_{1} x_{1} x_{1} x_{2} x_{2} x_{2} x_{2} \ldots: 0 . x_{1} x_{3} x_{5} \ldots \leq 0 . x_{2} x_{4} x_{6} \ldots\right.$ and $x_{2 k-1} \neq x_{2 k}$, for some $\left.k\right\}$;

- $A_{3}=\left\{[x, x]: x \in B_{2}\right\}$;

- $B_{3}=f\left(A_{3}\right)=\left\{0 . x_{1} x_{1} x_{1} x_{1} x_{1} x_{1} x_{1} x_{1} x_{2} x_{2} x_{2} x_{2} x_{2} x_{2} x_{2} x_{2} \ldots: 0 . x_{1} x_{3} x_{5} \ldots \leq\right.$ $0 . x_{2} x_{4} x_{6} \ldots$ and $x_{2 k-1} \neq x_{2 k}$, for some $\left.k\right\}$.

In general, for all $n \geq 1$, the set $B_{n}$ is constituted by the elements 0. $\underbrace{x_{1} \ldots x_{1}}_{2^{n}} \underbrace{x_{2} \ldots x_{2}}_{2^{n}} \underbrace{x_{3} \ldots x_{3}}_{2^{n}} \ldots$ such that $0 . x_{1} x_{3} x_{5} \ldots \leq 0 . x_{2} x_{4} x_{6} \ldots$ and $x_{2 k-1} \neq$ $x_{2 k}$, for some $k$. So, for $n \geq 1$, the elements belonging to $A_{n}$ have the form $[0 . \underbrace{x_{1} \ldots x_{1}}_{2^{n-1}} \underbrace{x_{2} \ldots x_{2}}_{2^{n-1}} \underbrace{x_{3} \ldots x_{3}}_{2^{n-1}} \ldots, 0 . \underbrace{x_{1} \ldots x_{1}}_{2^{n-1}} \underbrace{x_{2} \ldots x_{2}}_{2^{n-1}} \underbrace{x_{3} \ldots x_{3}}_{2^{n-1}} \ldots]$, being $0 . x_{1} x_{3} x_{5} \ldots \leq 0 . x_{2} x_{4} x_{6} \ldots$ and $x_{2 k-1} \neq x_{2 k}$, for some $k$. Thus, taking $[x, x]$, with $x=0.3321110 \ldots$, we have $[x, x] \notin A_{0}$. Besides, since there is not repetitions in the decimal expansion of $x,[x, x] \notin A_{n}$ for all $n \geq 2$. Finally, note that $0 . x_{1} x_{3} x_{5} \ldots=0.3210 \ldots$ and $0 . x_{2} x_{4} x_{6} \ldots=0.3110 \ldots$, so $0 . x_{1} x_{3} x_{5} \ldots \not \leq$ $0 . x_{2} x_{4} x_{6} \ldots$, which means that $[x, x] \notin A_{1}$. Thus, $[x, x] \in[0,1] \backslash \bigcup_{n \in \mathbb{N}} A_{n}$. Now, take $[y, y]=[0.332110 \ldots, 0.3321110] \in A_{0} \Rightarrow[y, y] \in \bigcup_{n \in \mathbb{N}} A_{n}$. Note that $[y, y]<_{2}[x, x]$. Following the proof of Cantor-Schroder-Bernstein theorem, we have $H([x, x])=0.3321110 \ldots$ and $H([y, y])=0.3333221111010 \ldots$, so $H([y, y])>H([x . x])$.

The order $\leq_{H}$, defined by $[a, b] \leq_{H}[c, d] \Leftrightarrow H([a, b]) \leq H([c, d])$ is a total order and is isometric to the usual order in $[0,1]$, being $H$, itself, the order isomorphism.

With the functions $g(t)=[0, t]$ or $g(t)=[t, 1]$, the function $H$ is not an order preserving bijection either. 
Although the function $H$ above is not an order preserving bijection, it coincides with $f$ except in a negligible set in the following sense. Identifying $\mathbb{I}[0,1]$ with the set $\mathbb{K}[0,1]=\left\{([x, y]) \in \mathbb{R}^{2}: 0 \leq x \leq y \leq 1\right\}$ (the upper part to the diagonal of the unit square), the subset of $\mathbb{K}[0,1]$ where $H$ is different from $f$ is a subset of the diagonal, which have null Lebesgue measure.

About a possible admissible order $\preceq$ such that $\langle\mathbb{I}[0,1], \preceq\rangle$ is isomorphic to $\langle[0,1], \leq\rangle$, the isomorphism $h: \mathbb{I}[0,1] \longrightarrow[0,1]$ can be seen as a function $h^{*}$ : $\mathbb{K}[0,1] \longrightarrow[0,1]$ and that function cannot be continuous, since continuous functions preserve connected sets and if one excludes both a point $a$ in $(0,1)$ and its pre-image $A \in \mathbb{K}[0,1]$, then the function $h^{* *}: \mathbb{K}[0,1] \backslash\{A\} \longrightarrow$ $[0,1] \backslash\{a\}$ is a continuous function sending the connected set $\mathbb{K}[0,1] \backslash\{A\}$ to the non-connected set $[0,1] \backslash\{a\}$.

\section{Final Remarks}

In this paper, we generalized the construction of admissible orders based on two aggregation functions and the lexicographical order by extending it to an arbitrary admissible order, instead of the lexicographical and functions that are not necessarily aggregation functions. We present an application of these new admissible orders on the decision making process where changes the order resulted in different choices. Our second contribution was to start investigating the existence of admissible orders on $\mathbb{I}[0,1]$ that are isomorphic to $[0,1]$ with its usual order. In Figure 1 we present, graphically, a summary of the results contained in this paper as well as the remaining open problem related to this question. In that figure, $\mathfrak{T}, \mathfrak{T}_{C}, \mathfrak{T}_{N C}$ and $\mathfrak{T}_{A}$ denote, respectively, the sets of total orders on $\mathbb{I}[0,1]$, total orders on $\mathbb{I}[0,1]$ with a continuous isomorphism to $\langle[0,1], \leq\rangle$ (this set is empty), total orders on $\mathbb{I}[0,1]$ with a non-continuous isomorphism to $\langle[0,1], \leq\rangle$, and admissible total orders on $\mathbb{I}[0,1]$.

In addition, although of the contributions of this paper are not relates with fuzzy intervals, in the sense of $[12,17]$, it may be a first step to get admissible ordering between fuzzy intervals, but it will be matter of a future research. In a future work, we intend to study new methods for constructing admissible orders on $\mathbb{I}[0,1]$, which are not necessarily based on aggregation functions and an order. Besides, we intend to give a full and definitive answer to the conjecture"If $\preceq$ is an admissible order on $\mathbb{I}[0,1]$, then $\langle\mathbb{I}[0,1], \preceq$,$\rangle is$ not isomorphic to $\langle[0,1], \leq\rangle$ ". 


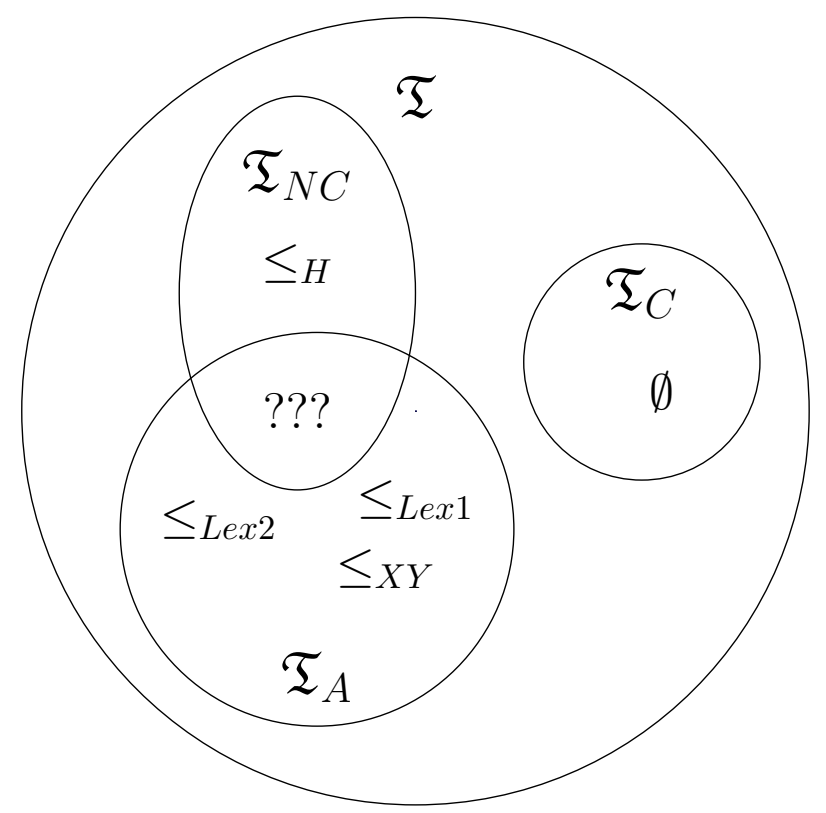

Figure 1: Sumary of isomorphism problem between $\mathbb{I}[0,1]$ with total (admissible) order and $[0,1]$ with their usual order.

\section{Acknowledgements}

This work was partially supported by the Brazilian funding agency CNPq (Conselho Nacional de Desenvolvimento Científico e Tecnológico), under Proc. No. 307781/2016-0 and by research project TIN2016-77356-P(AEI/FEDER,UE) of the Spanish Government.

\section{References}

[1] M.J. Asiaín, H. Bustince, R. Mesiar, A. Kolesárová, Z. Takáč, Negations with respect to admissible orders in the interval-valued fuzzy set theory, IEEE Trans. Fuzzy Syst., 26(2), 556-568, 2017.

[2] T.C. Asmus, G.P. Dimuro, B. Bedregal, On two-player interval-valued fuzzy Bayesian games, Int. J. Intell. Syst., 32(6), 557-596, 2017.

[3] E. Barrenechea, J. Fernandez, M. Pagola, F. Chiclana, H. Bustince, Construction of interval-valued fuzzy preference relations from ignorance 
functions and fuzzy preference relations. Application to decision making, Knowledge-Based Systems, 58, 33-44, 2014.

[4] U. Bentkowska, H. Bustince, A. Jurio, M. Pagola, B. Pekala, Decision making with interval-valued fuzzy preference relation and admissible orders, Applied Soft Computing, 35, 792-801, 2015.

[5] F. Bernstein, Untersuchungen aus der Mengenlehre, InauguralDissertation, George-Augustus-Universität, Güttingen, 1901.

[6] F. Bernstein, Sur la théorie des ensembles, C. R. Hebdomadaires Séances Acad. Sci., 143, 953-955, 1906.

[7] E. Borel. Leçons sur la théorie des fonctions. Gauthier-Villars, 1898.

[8] H. Bustince, E. Barrenechea, M. Pagola, J. Fernandez, Interval-valued fuzzy sets constructed from matrices: application to edge detection, Fuzzy Sets and Systems, 160, 1819-1840, 2009.

[9] H. Bustince, J. Fernandez, A. Kolesárová, R. Mesiar, Generation of linear orders for intervals by means of aggregation functions, Fuzzy Sets and Systems, 220, 69-77, 2013.

[10] H. Bustince, M. Galar, B. Bedregal, A. Kolesárová, R. Mesiar, A new approach to interval-valued Choquet integrals and the problem of ordering in interval-valued fuzzy set applications, IEEE Trans. Fuzzy Syst., 21(6), 1150-1162, 2013.

[11] G. Cantor, Beiträge zur Begründung der transfiniten Mengenlehre, Math. Ann., 46, 481-512, 1895.

[12] Y. Chalco-Cano, H. Román-Flores, F. Gomide, A new type of approximation for fuzzy intervals, Fuzzy Sets and Systems, 159(111), 1376-1383, 2008.

[13] J. Dugundij, Topology, Allyn and Bacon, 1966.

[14] M. Galar, J. Fernandez, G. Beliakov, H. Bustince, Interval-valued fuzzy sets applied to stereo matching of color images, IEEE Trans. Image Process., 20(7), 1949-1961, 2011. 
[15] A. Hinkis. Proofs of the Cantor-Bernstein Theorem: A Mathematical Excursion. Birkhäuser, 2013.

[16] E. Hüllermeier, K. Brinker, Learning valued preference structures for solving classification problems, Fuzzy sets and Systems, 159(18), 2337$2352,2008$.

[17] S.G. Li, Fuzzy intervals, Applied Mathematics Letters, 14(6), 737-740, 2001.

[18] L. Lima, B. Bedregal, H. Bustince, E. Barrenechea, M.P. da Rocha, An interval extension of homogeneous and pseudo-homogeneous t-norms and t-conorms, Inform. Sci., 355-356, 328-347, 2016.

[19] S. Nicolay, L. Simons, Bulding Cantor's bijection, arxiv:1409.1755v1 [math.HO].

[20] E.S. Palmeira, B. Bedregal, R. Mesiar, J. Fernandez, A new way to extend t-norms, t-conorms and negations Fuzzy Sets and Systems, 240, 1-21, 2014.

[21] B. Pang, Z. Xiu, Lattice-valued interval operators and its induced lattice-valued convex structures, IEEE Trans. Fuzzy Syst., 26(3), 15251534, 2018.

[22] D. Paternain, A. Jurio, E. Barrenechea, H. Bustince, B. Bedregal, E. Szmidt, An alternative to fuzzy methods in decision-making problems, Expert Syst. Appl., 39(9), 7729-7735, 2012.

[23] M. Reichbach, Une simple démonstration du théoréme de CantorBernstein, Colloq. Math., 3, 163, 1955.

[24] W. Rudin, Principles of Mathematical Analysis, McGraw-Hill, Inc., Third Edition, 1976.

[25] F. Santana, R. Santiago, A generalized distance based on a generalized triangle inequality, Inform. Sci., 345, 106-115, 2016.

[26] R. Santiago, B. Bedregal, B. Acióly, Formal aspects of correctness and optimality of interval computations, Form. Asp. Comput., 18(2), 231243, 2006 . 
[27] J. Sanz, A. Fernandez, H. Bustince, F. Herrera, A genetic tuning to improve the performance of fuzzy rule-based classification systems with interval-valued sets: degree of ignorance and lateral position, Internat. J. Approx. Reason., 52(6), 751-766, 2011.

[28] E. Schröder, Über zwei Definitionen der Endlichkeit und G. Cantor'sche Sätze, Abh. der Kaiserl. Leop.-Carol Deutchen Akademie der Naturforsher, 71(6), 301-362, 1898.

[29] L. Silva, R. Moura, A. Canuto, R. Santiago, B. Bedregal, New ways to calculate centers for interval data in fuzzy clustering algorithms, In Proc. 2014 IEEE Int. Conf. Fuzzy Syst., Beijing, China, 2014.

[30] L. Silva, R. Moura, A. Canuto, R. Santiago, B. Bedregal, An intervalbased framework for fuzzy clustering applications, IEEE Trans. Fuzzy Syst., 23(6), 2174-2187, 2015.

[31] A. Tarski, A lattice-theoretical fixpoint theorem and its applications, Pacific J. Math., 5(2), 285-309, 1955.

[32] Z. Xu, R. Yager, Some geometric aggregation operators based on intuitionistic fuzzy sets, Int. J. Gen. Syst., 35(4), 417-433, 2006.

[33] L. Zadeh, The concept of a linguistic variable and its applications to approximate reasoning, Inform. Sci., Part I 8(3), 199-249, Part II 8(4), 301-357, Part III 9(1), 43-80, 1975.

[34] H. Zapata, H. Bustince, S. Montes, B. Bedregal, G.P. Dimuro, Z. Takáć, M. Baczyński, J. Fernandez, Interval-valued implications and intervalvalued strong equality index with admissible orders, Internat. J. Approx. Reason., 88, 91-109, 2017. 